\title{
Impact of body mass index levels on lipid abnormalities in Chinese Asians, American Blacks and American Whites: The People's Republic of China (PRC) and Atherosclerosis Risk in Communities (ARIC) Studies
}

\author{
Kimberly P Truesdale ${ }^{a}$, June Stevens ${ }^{a, b}$, and Jianwen Cai ${ }^{c}$ \\ aDepartment of Nutrition, Gillings School of Global Public Health, University of North Carolina, \\ Chapel Hill, North Carolina, United States \\ bDepartment of Epidemiology, Gillings School of Global Public Health, University of North \\ Carolina, Chapel Hill, North Carolina, United States \\ 'Department of Biostatistics, Gillings School of Global Public Health, University of North Carolina, \\ Chapel Hill, North Carolina, United States
}

\section{Abstract}

Background-Several researchers have reported that Chinese adults may have a greater chronic disease burden than Whites, especially at lower body mass index (BMI) levels.

Objectives-To compare the incidence of lipid abnormalities in Chinese $(n=5,303)$, White $(n=10,752)$ and Black $(n=3,408)$ middle-aged adults and the effect of BMI on these incidences.

\begin{abstract}
Methods-Data were from the People's Republic of China (PRC) and the Atherosclerosis Risk in Communities (ARIC) studies. In each ethnic group, we calculated the adjusted cumulative incidence for high total cholesterol ( $\geq 240 \mathrm{mg} / \mathrm{dL})$, LDL-cholesterol $(\geq 160 \mathrm{mg} / \mathrm{dL})$, and triglycerides ( $\geq 200 \mathrm{mg} / \mathrm{dL}$ ) and low HDL-cholesterol ( $\leq 40$ in men and $\leq 50 \mathrm{mg} / \mathrm{dL}$ in women) adjusted for age, gender, education, field site, smoking and drinking status. Risk differences associated with BMI (referent $=18.5-22.9 \mathrm{~kg} / \mathrm{m}^{2}$ ) were calculated using weighted linear regression and slopes compared using the Wald test.
\end{abstract}

\begin{abstract}
Results-Chinese had lower incidence of abnormal total cholesterol, LDL-cholesterol and triglycerides than Whites in most BMI groups and had lower incidence of abnormal HDLcholesterol and triglycerides than Blacks. Across the range of 18.5 to $<30$, BMI was more strongly associated with the incidence of having high total cholesterol in Chinese and Whites than in Blacks. Similar trends were seen for LDL-cholesterol and triglycerides, but were not always statistically significant. In contrast, BMI was more highly associated with incidence of low HDLcholesterol in Whites than in Chinese or Blacks.
\end{abstract}

Conclusion-Although differences in the incidence of lipid abnormalities and the impact of BMI were identified, results varied by lipid type indicating no consistent ethnic/national pattern.

(C) 2011 Elsevier Ireland Ltd. All rights reserved.

Corresponding author: Dr. Kimberly P Truesdale, Department of Nutrition, CB 7461, University of North Carolina, Chapel Hill, NC 27599, USA, Phone: 919-966-2327, FAX: 919-966-7215, Kim_Truesdale@unc.edu.

Publisher's Disclaimer: This is a PDF file of an unedited manuscript that has been accepted for publication. As a service to our customers we are providing this early version of the manuscript. The manuscript will undergo copyediting, typesetting, and review of the resulting proof before it is published in its final citable form. Please note that during the production process errors may be discovered which could affect the content, and all legal disclaimers that apply to the journal pertain. 


\section{Keywords}

Obesity; Total cholesterol; LDL-cholesterol; HDL-cholesterol; Triglycerides; Ethnicity

\section{INTRODUCTION}

Data from the 2002 Chinese Nutrition and Health Survey indicated that $22.9 \%$ of Chinese adults 45-59 years of age had dyslipidemia (high total cholesterol, high triglycerides or low HDL-cholesterol) [1]. High triglycerides were the most common (15.9\%) lipid abnormality. In contrast, approximately $48 \%$ of US adults had at least one lipid abnormality (high LDLcholesterol, high triglycerides or low HDL-cholesterol) with high LDL-cholesterol being the most common (28\%) [2]. A larger percentage of Americans 45-54 and 55-64 years of age meet the Adult Treatment Panel III guideline recommendation for lifestyle change (39.8\% and $54.1 \%$ ) or drug therapy (19.6\% and $29.7 \%$ ) compared to the percentage of Chinese adults in the same age categories (18.0\% and $27.7 \%$ for lifestyle change and $6.8 \%$ and $11.8 \%$ for drug therapy), respectively [3]. However, due to the differences in population size the absolute number of adults who need either lifestyle change or drug therapy is higher in China than the United States.

Researchers have shown unfavorable changes in lipid levels as body mass index (BMI) increases in Asian and non-Asian populations. However, there is still debate whether the impact of BMI on lipid levels or dyslipidemia is greater among Asians compared to Caucasian (or non-Asian) populations. Many of the studies that concluded a greater impact among Asians did not include a Caucasian comparison group [4-7]. The limited number of studies that have included a Caucasian or non-Asian comparison found a greater effect of BMI on lipid levels and/or dylipidemia/lipid abnormalities [8-11]. To our knowledge, no study has longitudinally examined the effects of BMI on incident lipid abnormalities across ethnic groups. The objectives of this study were to determine and compare: 1) the incidence of lipid abnormalities in Chinese Asian, American White and American Black middle-aged adults, and 2) the effect of BMI on incident lipid abnormalities in each ethnic group.

\section{MATERIALS AND METHODS}

\section{Study populations}

Data are from two prospective cohort studies - the People's Republic of China (PRC) Study and the Atherosclerosis Risk in Communities (ARIC) Study. The PRC study examined Chinese Asian women and men living in urban and rural Beijing (northern China) and Guangzhou (southern China) [12]. Baseline data were collected in 1983-1984 with followup examinations in 1987-88 and 1993-94. The ARIC study examined American White and American Black men and women in four United States communities: Forsyth County, North Carolina, Jackson, Mississippi, the northwestern suburbs of Minneapolis, Minnesota and Washington County, Maryland [13]. Baseline data were collected in 1987-89 with followup examinations in 1990-1992, 1993-1995 and 1996-1999. Since participants from the PRC study only had two follow-up examinations, we elected to use only two follow-up examinations in ARIC therefore data from the third ARIC examination (1993-1995) was not included in the analysis. The follow-up period was 8.2 years for Chinese Asians and American Whites and 7.9 years for American Blacks.

Only participants aged 45 to 64 at baseline from the PRC study $(n=6,575)$ were included in the analysis. This age range was select in order to match the baseline age range of participants from the ARIC study. For the ARIC cohort, we excluded Blacks from Washington County, Maryland or Minneapolis, Minnesota $(\mathrm{n}=55)$ and participants who did 
not classify their race/ethnicity as White or Black $(n=48)$ because they were too small in number to allow ethnic and field center-specific analyses. For both studies, participants were excluded if they were missing baseline BMI $(n=58)$, both follow-up visits $(n=1,760)$, all baseline lipid measures $(n=884)$, or pertinent covariates $(n=99)$. The analysis sample included 5,303 Chinese Asians, 10,752 American Whites and 3,408 American Blacks. These studies were approved by the Institutional Review Boards (IRB) at each field center and this analysis was approved by the University of North Carolina at Chapel Hill (UNC) Public Health IRB on research involving human subjects.

\section{Measurements}

The data collection protocols and training manuals for all measurements were developed by the Collaborative Studies Coordinating Center (CSCC) at the UNC. These protocols were very similar between the studies. In addition, the CSCC handled the review, processing and analyses of all data for both ARIC and PRC studies. In both studies, height was measured without shoes to the nearest centimeter $(1$ centimeter $=0.394 \mathrm{inch})$ using a metal rule attached to a wall and a standard triangular headboard using a vertical ruler. Body weight was measured to the nearest pound ( 1 pound $=0.454$ kilogram $)$ in light clothing $(\mathrm{PRC}$ cohort) or scrub suit (ARIC cohort) using a beam balance scale. Baseline BMI was calculated as weight in kilograms divided by height in meters squared then categorized into seven mutually exclusive categories ( $>18.5, \geq 18.5$ to $<23.0, \geq 23.0$ to $<25.0, \geq 25.0$ to $<27.5$, $\geq 27.5$ to $<30.0, \geq 30.0$ to $<32.5, \geq 32.5 \mathrm{~kg} / \mathrm{m}^{2}$ ) [14].

Education, smoking status and alcohol consumption were assessed using interviewer administered questionnaires. Since educational attainment (as measured in years) was not comparable in the United States and China, we created cohort specific education categories (low, medium, high). In ARIC, participants were categorized as less than high school graduate, high school graduate, or at least some college. In PRC, participants were categorized as less than primary education, primary education, or at least secondary education. We dichotomized participants as current smoker (cigarettes or leaf products) or non-smoker and current alcoholic beverage drinker or non-drinker.

Study participants were instructed to fast for 12 hours prior to their clinic visit. Fasting blood were sent to laboratories in Beijing or Guangzhou, China (PRC) or in Houston, Texas (ARIC). All laboratories were standardized according to the Centers for Disease Control and Prevention protocol. Total cholesterol [15] and triglycerides [16] were determined by enzymatic methods. HDL-cholesterol was measured after dextran-magnesium precipitation [17]. The Friedewald method was used to calculate LDL-cholesterol [18]. LDL-cholesterol was not calculated for participants with triglyceride levels $\geq 400 \mathrm{mg} / \mathrm{dL}$. Participants with abnormal total cholesterol ( $\geq 240 \mathrm{mg} / \mathrm{dL})$, LDL-cholesterol ( $\geq 160 \mathrm{mg} / \mathrm{dl})$, HDL-cholesterol $(\leq 40 \mathrm{mg} / \mathrm{dL}$ in men and $\leq 50 \mathrm{mg} / \mathrm{dL}$ in women), and triglycerides ( $\geq 200 \mathrm{mg} / \mathrm{dL})$ at baseline were excluded for the respective incidence analysis. If a participant had abnormal total cholesterol, LDL-cholesterol, HDL-cholesterol or triglycerides at either follow-up visit then they were classified as an incident case for that respective analysis. Lipid lowering medication usage was not available for the Chinese, therefore it was not used to define lipid abnormalities.

\section{Statistical analyses}

Adjusted lipid abnormalities incidence rates over approximately 8 years were calculated separately for each ethnic group using logistic regression (PROC LOGISTIC) in SAS, version 9.1 (Cary, North Carolina). This approach was selected after log binomial (with log link), binomial models (with identity link) and poisson models did not converge for several outcomes. The models were adjusted for gender, age, smoking status, alcohol consumption, 
education and field center. To accommodate our focus on risk differences rather than ratios we used the same, pre-specified level or distribution for gender (54\% female), age (53.2 years), smoking status (non-smoker) and alcohol consumption (non-drinker) in each race group. Because educational levels were widely divergent in the two cohorts, the weighted cumulative incidences were based on the ethnic-specific distribution. Similarly, field centers were specific to the ethnic groups. The delta method [19] was used to calculate standard errors and $95 \%$ confidence intervals for weighted cumulative incidences and risk differences. For each BMI category, the weighted cumulative incidences were compared across pairs (Chinese versus Whites, Chinese versus Blacks, and Whites versus Blacks) using the Wald statistics with one degree of freedom.

To summarize the risk difference results across BMI categories, we constructed weighted least squares regression models using the adjusted risk differences as the dependent variables and the ethnic specific median BMI within each category as independent variables. The weights used were the inverse of the estimated variances for the adjusted risk differences. Results from this analysis were also compared among ethnic group pairs using the Wald statistics with one degree of freedom.

\section{RESULTS}

Americans from the ARIC cohort were slightly older than the Chinese Asians in the PRC study (Table 1). As expected, the mean BMI among Chinese was smaller $\left(22.4 \mathrm{~kg} / \mathrm{m}^{2}\right)$ compared to mean BMI among Whites $\left(27.0 \mathrm{~kg} / \mathrm{m}^{2}\right)$ and Blacks $\left(29.6 \mathrm{~kg} / \mathrm{m}^{2}\right)$. Less than 3.0 percent of Chinese were obese $\left(\geq 30.0 \mathrm{~kg} / \mathrm{m}^{2}\right)$, whereas less than one percent of Americans were underweight $\left(<18.5 \mathrm{~kg} / \mathrm{m}^{2}\right)$. Chinese were more likely than Blacks and Whites to be current smokers but less likely to consume alcoholic beverages or have "high" education attainment. At baseline, low HDL-cholesterol was the most prevalent type of lipid abnormality in all ethnic groups.

We did not find significant interactions between gender and BMI categories for the four outcomes; therefore we do not show analyses by gender. The adjusted cumulative incidence for high total cholesterol, high LDL-cholesterol, low HDL-cholesterol, and high triglycerides across BMI categories are shown in figures $1 \mathrm{a}-1 \mathrm{~d}$, respectively. Incidence rates were not calculated for obese Chinese or underweight Americans due to small sample sizes. Across the BMI categories examined, Whites were significantly more likely to develop high total cholesterol than Chinese (Figure 1a). Blacks had lower incidence rates of high total cholesterol than Whites, although not always statistical significant. In contrast, Blacks had slightly higher (not significant) incidence rates than Chinese.

In the two normal weight categories, Whites had significantly higher incidence rates of high LDL-cholesterol than Chinese (Figure 1b). This trend continued in the higher BMI categories, although not statistically significant. The incidence rates of high LDL-cholesterol among Blacks were slightly higher than Chinese and slightly lower than Whites in the normal and overweight BMI categories. Obese Blacks had higher incidence rates of high LDL-cholesterol than Whites.

When examining the incidence of low HDL-cholesterol, we found similar incidence rates between Whites and Chinese with one exception (Figure 1c). In the $\geq 27.5-<30.0 \mathrm{~kg} / \mathrm{m}^{2}$ category, the incidence was significantly higher among Whites than Chinese. Both Whites and Chinese had significantly higher incidence rates of low HDL-cholesterol than Blacks in all BMI categories examined except the $\geq 23.0$ to $<25.0 \mathrm{~kg} / \mathrm{m}^{2}$ category.

Whites had the highest incidence of high triglycerides across all BMI categories (Figure 1d). Chinese tended to have slightly lower incidence rates than Whites, although not always 
statistically significant. Both Whites and Chinese had significantly higher incidence rates than Blacks.

The adjusted risk differences for the four outcomes by ethnicity are shown in table 2 . In general, the risk differences in the BMI categories above the referent group $(\geq 18.5-<23.0$ $\mathrm{kg} / \mathrm{m}^{2}$ ) were positive and tended to increase with increasing BMI. Nevertheless, the risk difference confidence intervals were wide and no BMI category differences within ethnicity.

Figures 2a-d compares the effect of BMI (as measured by the slope of the risk difference) on lipid abnormalities by ethnicity. The slopes were calculated using the full BMI range and the restricted BMI range ( $\geq 18.5$ to $\left.<30.0 \mathrm{~kg} / \mathrm{m}^{2}\right)$ in which all three ethnic groups had sufficient data to allow stable estimates. Statistically significant differences between pairs of estimates are indicated in the figures.

For total cholesterol (Figure 2a) the slope was steeper in Chinese than Whites and Blacks when the entire range was examined. However, using the restricted BMI range we found a slightly steeper slope in both Chinese and Whites compared to Blacks. These trends were similar for LDL-cholesterol (Figure 2b). For low HDL-cholesterol (Figure 2c) we found the effect of BMI was similar in Chinese and Whites and stronger than in Blacks when the full BMI range was examined. In the restricted BMI range, the BMI association with HDLcholesterol was strongest in the Whites and tended to be weakest in Blacks. For triglycerides, associations were similar in Chinese and Whites (Figure 2d) when examined in both the full range and the restricted range of BMI. Associations between BMI and triglycerides were weakest in Blacks although confidence intervals overlapped with the Chinese in the restricted analysis.

\section{DISCUSSION}

In this study, we examined the impact of baseline BMI on risk differences rather than risk ratios. Stevens et al. demonstrated how comparisons of BMI risk across ethnic groups are strongly influenced by the metrics compared if the incidence of the outcomes studied were different in the reference category [20]. In those circumstances, risk differences are more relevant than risk ratios. Here, in the reference BMI range $\left(\geq 18.5-<23.0 \mathrm{~kg} / \mathrm{m}^{2}\right)$ the incidence of high total cholesterol and LDL-cholesterol was lower in Chinese than in Whites $(\mathrm{p}<0.05)$ and Blacks (not significant). However, for HDL-cholesterol and triglycerides, incidence rates were similar in Chinese and Whites, and lowest in the Blacks $(\mathrm{p}<0.05)$. Although these differences were not always statistically significant, they do indicate important trends in ethnic differences among adults with a healthy weight.

Comparisons of the impact of elevated BMI across ethnicities was complicated in this analysis by the very different BMI distributions, with few obese Chinese and underweight Americans. Therefore, we examined BMI within a restricted range in which all groups had adequate representation to provide a more unbiased comparison among ethnicities. Across that range $\left(18.5-30 \mathrm{~kg} / \mathrm{m}^{2}\right)$ BMI was more strongly associated with the incidence of having high total cholesterol in Chinese and Whites than in Blacks. Similar trends were seen for LDL-cholesterol and for triglycerides, but were not always statistically significant. In contrast, BMI was more highly associated with incidence of low HDL-cholesterol in Whites than in Chinese or Blacks.

To our knowledge, no other study has compared the baseline BMI-lipid relationship between Chinese and Blacks, however, we know of 8 studies [3,8-11,21-23] that compared the effect of BMI on lipid levels, dyslipidemia or lipid abnormalities in Asian and non-Asian/ Caucasian adults. All of these studies were cross-sectional and the findings were mixed. Kesteloot et al. found Belgian men and women had higher total cholesterol than Koreans 
and Chinese [21]. For HDL-cholesterol, there was no difference between Belgian and Chinese men but higher levels among Belgian women compared to Chinese and Korean women. Iwao et al. found Whites had higher mean BMI levels but lower prevalence of abnormal total cholesterol and LDL-cholesterol levels than Japanese [8]. They found similar prevalence of abnormal triglycerides between Caucasian and Japanese men and higher prevalence of abnormal HDL-cholesterol among Whites. Neither study examined the impact of BMI or compared groups with similar levels of BMI.

Lear et al. found similar BMI levels between adults of European and South Asian descent living in Canada [22]. However, South Asian men had higher mean total cholesterol, LDLcholesterol, triglycerides and lower mean HDL-cholesterol than men of European decent. In women, South Asians had lower HDL-cholesterol and higher triglycerides but similar total and LDL-cholesterol than women of European descent. After adjusting for BMI, ethnicity was an independent predictor of all lipid outcomes.

Two studies have used receiver operating characteristic (ROC) curves to estimate the BMI associated with the greatest sum of sensitivity and specificity $(\Sigma \mathrm{SS})$ to identify dyslipidemia $[9,10]$. Both studies calculated $\Sigma$ SS to be at a smaller BMI in Chinese/Asians than European/non-Asians. However, sensitivity and specificity are strongly influenced by the BMI distribution which varied across ethnicities. Therefore it is difficult to ascertain how the levels of risk associated with BMI may have differed in these groups.

CVD risk factors and prevalence vary across Asians [6,9] and between Whites living in Europe and North America [8,22,23]. Therefore, the differences in the findings between our study and previous studies could be influenced by where the subjects lived or the specific Asian ethnic group examined. Chandalia et. al found vast differences in the frequency of low HDL-cholesterol among lean women from rural India (72\%) and urban India (56\%) compared to Asian Indians in US (48\%) and Whites (25\%) [11]. Similar disparities were observed among men $(52 \%, 42 \%, 28 \%$ and $35 \%$, respectively).

The current study has several limitations. Although models were adjusted for cigarette smoking and education, these variables may mean different things in China and the US. For example, the content and concentrations of the chemicals in cigarettes may differ and could impact lipids differently. Therefore, the incidence levels presented were calculated for nonsmokers. The ethnic-specific distribution of educational attainment (low, medium, high) was used to calculate the predicted incidence levels since it is plausible that high education in the US (college education) may not be equivalent to high education(post secondary) in China. Other unmeasured social and environmental covariates might have influenced the BMI-lipid relationships. Comparable diet and physical activity were not available from both studies, therefore, these variables were not included in the analysis. It is reasonable to assume that the diet and physical activity level varied between Chinese and Americans. Researchers have shown associations between these variables and lipid levels [24].

In the current study, ethnicity and nationality were confounded in all comparisons to Chinese. Although not as obvious as differences between China and the US, the environments of the White and Black participants were also likely to vary importantly. Interpretations across groups must be done with care and recognition that numerous aspects of the social, cultural, economic, geographic and political environments were likely different between groups. Therefore, differences found here cannot be attributed to racial or environmental differences, but to a combination of these and other factors. Also, the cohorts were not selected to be nationally representative although the ARIC cohort represents 4 US communities. Nevertheless, ethnic comparisons are of interest, particularly since recommended public health action points for Asians [14] are lower than the recommended 
BMI cutpoints for overweight and obesity used in the US and globally (as recommended by the WHO) [25,26]. Information showing increased risk with increasing BMI in Chinese compared to Americans would support the utility of these different BMI cutpoints, albeit only for risk of lipid abnormalities in this work.

The current study has several strengths. The same coordinating center for was used for both the PRC and ARIC studies with similar data collection methods. Additionally, we compared Chinese adults living in China with two ethnic groups in the US. To our knowledge, all previous studies have been cross-sectional and only were able to examine prevalence.

Our research group has conducted similar analyses for diabetes and hypertension [27]. We found that Chinese had a higher incidence of hypertension and diabetes than Whites and Blacks. Additionally, the effect of BMI on hypertension was greater in Chinese than Whites and Blacks and the effect of BMI on diabetes was greater in Chinese and Blacks than Whites. The discrepancy in the results for hypertension, diabetes and lipid abnormalities underscores the fact that ethnic differences in risk factor patterns and the impact of BMI on those patterns may vary based on the particular risk factor studied. It may be prudent for investigators to refrain from making recommendations for a policy of different BMI cutpoints in different national or ethnic groups based on studies of risk factors, and instead to restrict the evidence base for such recommendations to studies that examined BMI associations with hard endpoints (e.g. stroke, heart disease, and total mortality).

\section{Acknowledgments}

The Atherosclerosis Risk in Communities (ARIC) Study is carried out as a collaborative study supported by National Heart, Lung, and Blood Institute contracts N01-HC-55015, N01-HC-55016, N01-HC-55018, N01HC-55019, N01-HC-55020, N01-HC-55021, and N01-HC-55022. The People's Republic of China (PRC) Study was carried out by the National Heart, Lung, and Blood Institute under contracts N01-HV-12243, N01-HV-08112, and N01-HV-59224 with the University of North Carolina, Chapel Hill, North Carolina, and the People's Republic of China Ministry of Public Health, the Cardiovascular Institute and Fu Wai Hospital, Chinese Academy of Medical Sciences, Beijing, and the Guangdong Provincial Cardiovascular Institute, Guangzhou. This research was supported in part by a grant (RR00046) from the General Clinical Research Centers' program of the Division of Research Resources, National Institutes of Health, and by grants (R01 DK069678 and P30 DK056350-09S2) from the National Institute of Diabetes and Digestive and Kidney Diseases.

The authors thank the staff of the ARIC Study and the staff of the PRC Study for their important contributions.

\section{REFERENCES}

1. Zhao WH, Zhang J, Zhai Y, et al. Blood lipid profile and prevalence of dyslipidemia in Chinese adults. Biomed Environ Sci. 2007; 20:329-335. [PubMed: 17948769]

2. Ghandehari H, Kamal-Bahl S, Wong ND. Prevalence and extent of dyslipidemia and recommended lipid levels in US adults with and without cardiovascular comorbidities: the National Health and Nutrition Examination Survey 2003-2004. Am Heart J. 2008; 156:112-119. [PubMed: 18585505]

3. Muntner P, Gu D, Reynolds RF, et al. Therapeutic lifestyle changes and drug treatment for high blood cholesterol in China and application of the Adult Treatment Panel III guidelines. Am J Cardiol. 2005; 96:1260-1265. [PubMed: 16253594]

4. Shiwaku K, Anuurad E, Enkhmaa B, et al. Overweight Japanese with body mass indexes of 23.024.9 have higher risks for obesity-associated disorders: a comparison of Japanese and Mongolians. Int J Obes Relat Metab Disord. 2004; 28:152-158. [PubMed: 14557832]

5. Li R, Lu W, Jia J, et al. Relationships between indices of obesity and its cardiovascular comorbidities in a Chinese population. Circ J. 2008; 72:973-978. [PubMed: 18503225]

6. Deurenberg-Yap M, Chew SK, Lin VF, et al. Relationships between indices of obesity and its comorbidities in multi-ethnic Singapore. Int J Obes Relat Metab Disord. 2001; 25:1554-1562. [PubMed: 11673781] 
7. Wildman RP, Gu D, Reynolds K, Duan X, He J. Appropriate body mass index and waist circumference cutoffs for categorization of overweight and central adiposity among Chinese adults. Am J Clin Nutr. 2004; 80:1129-1136. [PubMed: 15531658]

8. Iwao N, Iwao S, Muller DC, et al. Differences in the relationship between lipid CHD risk factors and body composition in Caucasians and Japanese. Int J Obes (Lond). 2005; 29:228-235. [PubMed: 15570315]

9. Razak F, Anand SS, Shannon H, et al. Defining obesity cut points in a multiethnic population. Circulation. 2007; 115:2111-2118. [PubMed: 17420343]

10. Barzi F, Woodward M, Czernichow S, et al. The discrimination of dyslipidaemia using anthropometric measures in ethnically diverse populations of the Asia-Pacific Region: the Obesity in Asia Collaboration. Obes Rev. 11:127-136. [PubMed: 19493299]

11. Chandalia M, Mohan V, Adams-Huet B, Deepa R, Abate N. Ethnic difference in sex gap in highdensity lipoprotein cholesterol between Asian Indians and Whites. J Investig Med. 2008; 56:574580.

12. An epidemiological study of cardiovascular and cardiopulmonary disease risk factors in four populations in the People's Republic of China. Baseline report from the P.R.C.-U.S.A. Collaborative Study. People's Republic of China--United States Cardiovascular and Cardiopulmonary Epidemiology Research Group. Circulation. 1992; 85:1083-1096. [PubMed: 1537106]

13. The Atherosclerosis Risk in Communities (ARIC) Study: design and objectives. The ARIC investigators. Am J Epidemiol. 1989; 129:687-702. [PubMed: 2646917]

14. Appropriate body-mass index for Asian populations and its implications for policy and intervention strategies. Lancet. 2004; 363:157-163. [PubMed: 14726171]

15. Siedel J, Hagele EO, Ziegenhorn J, Wahlefeld AW. Reagent for the enzymatic determination of serum total cholesterol with improved lipolytic efficiency. Clin Chem. 1983; 29:1075-1080. [PubMed: 6851096]

16. Nagele U, Hagele EO, Sauer G, et al. Reagent for the enzymatic determination of serum total triglycerides with improved lipolytic efficiency. J Clin Chem Clin Biochem. 1984; 22:165-174. [PubMed: 6716056]

17. Warnick GR, Benderson J, Albers JJ. Dextran sulfate-Mg2+ precipitation procedure for quantitation of high-density-lipoprotein cholesterol. Clin Chem. 1982; 28:1379-1388. [PubMed: 7074948]

18. Friedewald WT, Levy RI, Fredrickson DS. Estimation of the concentration of low-density lipoprotein cholesterol in plasma, without use of the preparative ultracentrifuge. Clin Chem. 1972; 18:499-502. [PubMed: 4337382]

19. Oehlert GW. A Note on the Delta Method. The American Statistician. 1992; 46:27-29.

20. Stevens J, Juhaeri, Cai J, Jones DW. The effect of decision rules on the choice of a body mass index cutoff for obesity: examples from African American and white women. Am J Clin Nutr. 2002; 75:986-992. [PubMed: 12036803]

21. Kesteloot H, Huang DX, Yang XS, et al. Serum lipids in the People's Republic of China. Comparison of Western and Eastern populations. Arteriosclerosis. 1985; 5:427-433. [PubMed: 4038156]

22. Lear SA, Toma M, Birmingham CL, Frohlich JJ. Modification of the relationship between simple anthropometric indices and risk factors by ethnic background. Metabolism. 2003; 52:1295-1301. [PubMed: 14564681]

23. Lyratzopoulos G, McElduff P, Heller RF, Hanily M, Lewis PS. Comparative levels and time trends in blood pressure, total cholesterol, body mass index and smoking among Caucasian and SouthAsian participants of a UK primary-care based cardiovascular risk factor screening programme. BMC Public Health. 2005; 5:125. [PubMed: 16313671]

24. Third Report of the National Cholesterol Education Program (NCEP) Expert Panel on Detection, Evaluation, and Treatment of High Blood Cholesterol in Adults (Adult Treatment Panel III) final report. Circulation. 2002; 106:3143-3421. [PubMed: 12485966] 
25. World Health Organization Consultation on Obesity. Obesity: Preventing and Managing the Global Epidemic: Report of a WHO Consultation on Obesity, 3-5 June 1997. Geneva: World Health Organization; 1998. p. 1-276.

26. World Health Organization Expert Committee. Physical status: The use and interpretation of anthropometry. Geneva: World Health Organization; 1995.

27. Stevens J, Truesdale KP, Katz EG, Cai J. Impact of body mass index on incident hypertension and diabetes in Chinese Asians, American Whites, and American Blacks: the People's Republic of China Study and the Atherosclerosis Risk in Communities Study. Am J Epidemiol. 2008; 167:1365-1374. [PubMed: 18375949] 
$1 a$

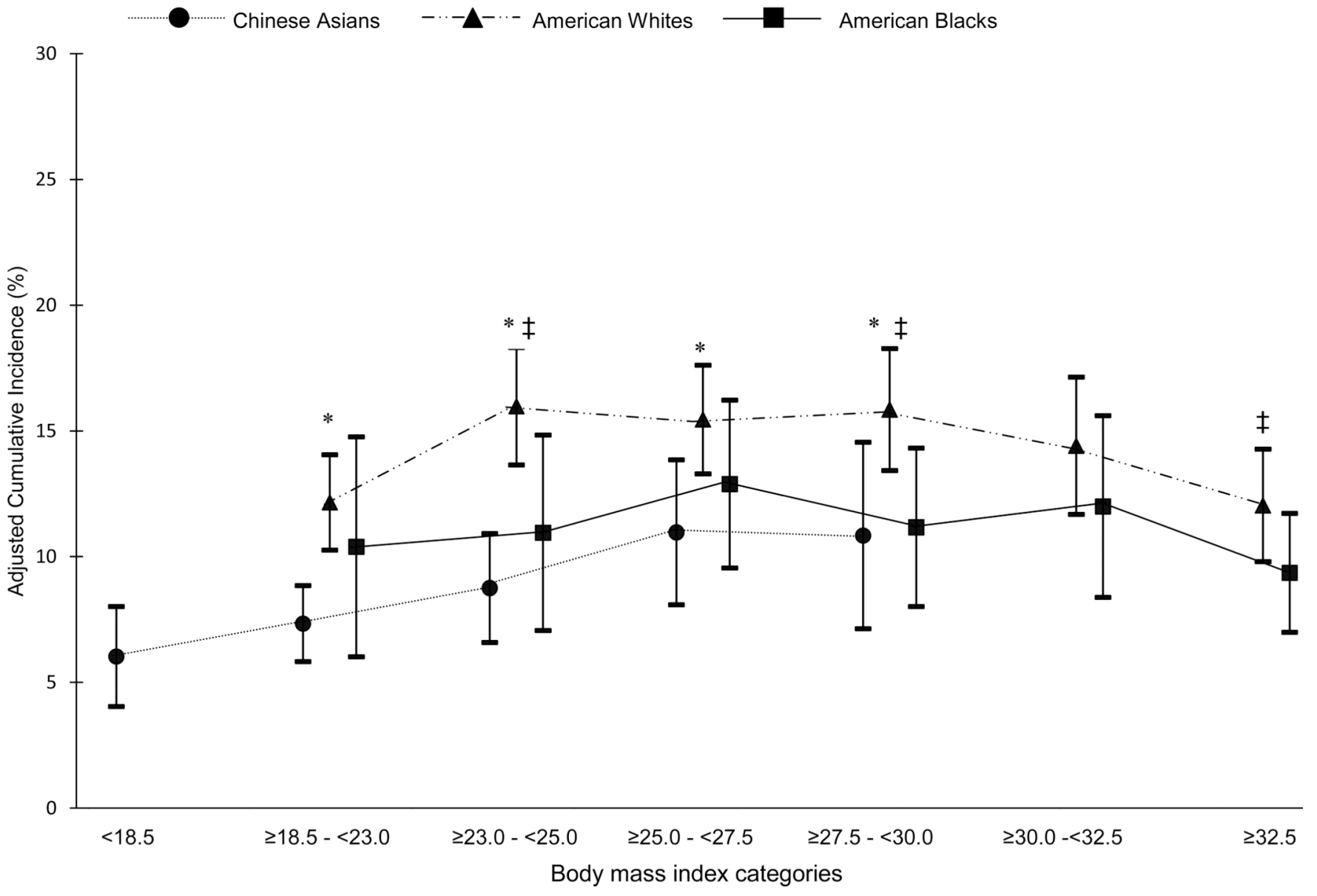

Atherosclerosis. Author manuscript; available in PMC 2012 October 1. 
1b

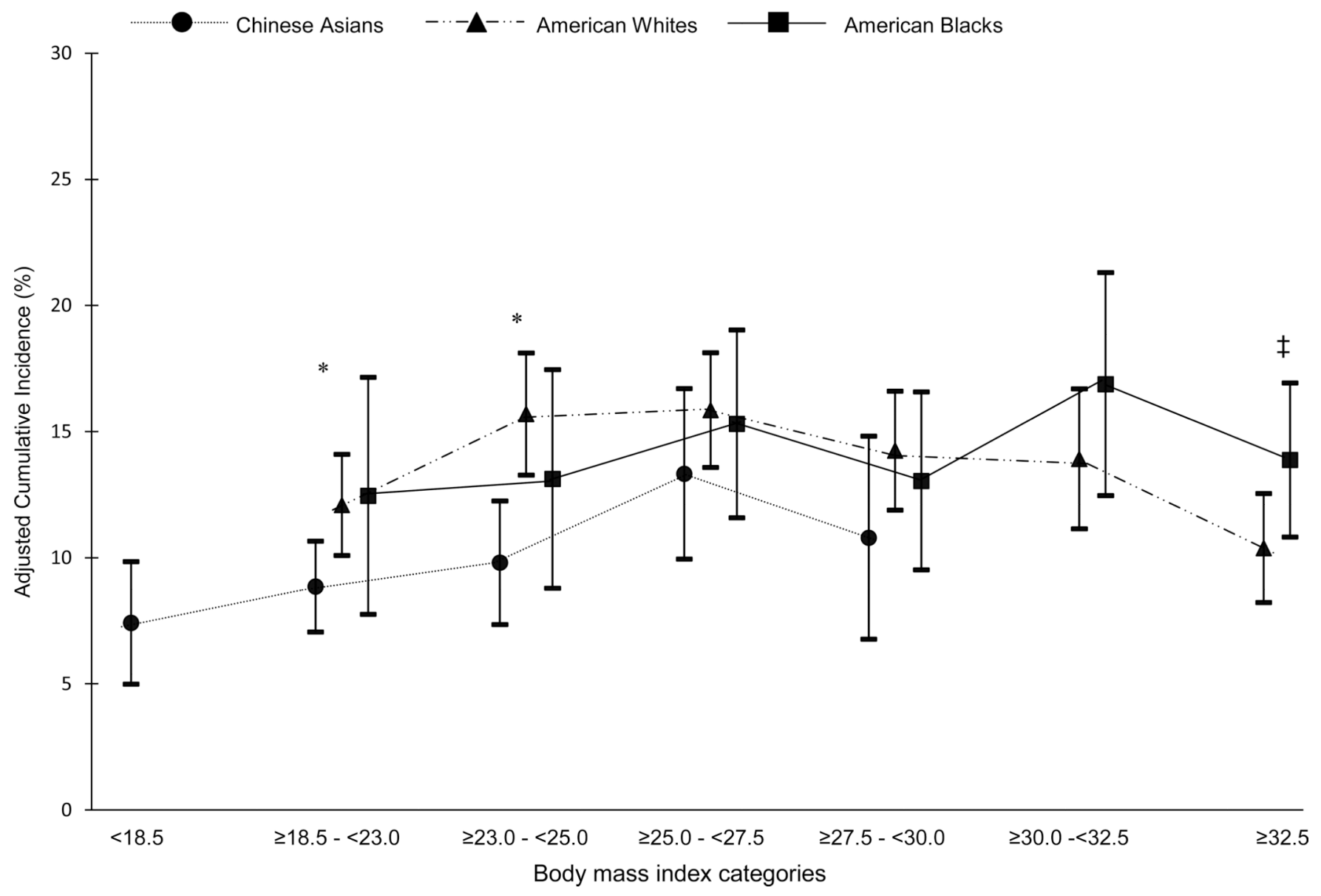

Atherosclerosis. Author manuscript; available in PMC 2012 October 1. 
$1 c$

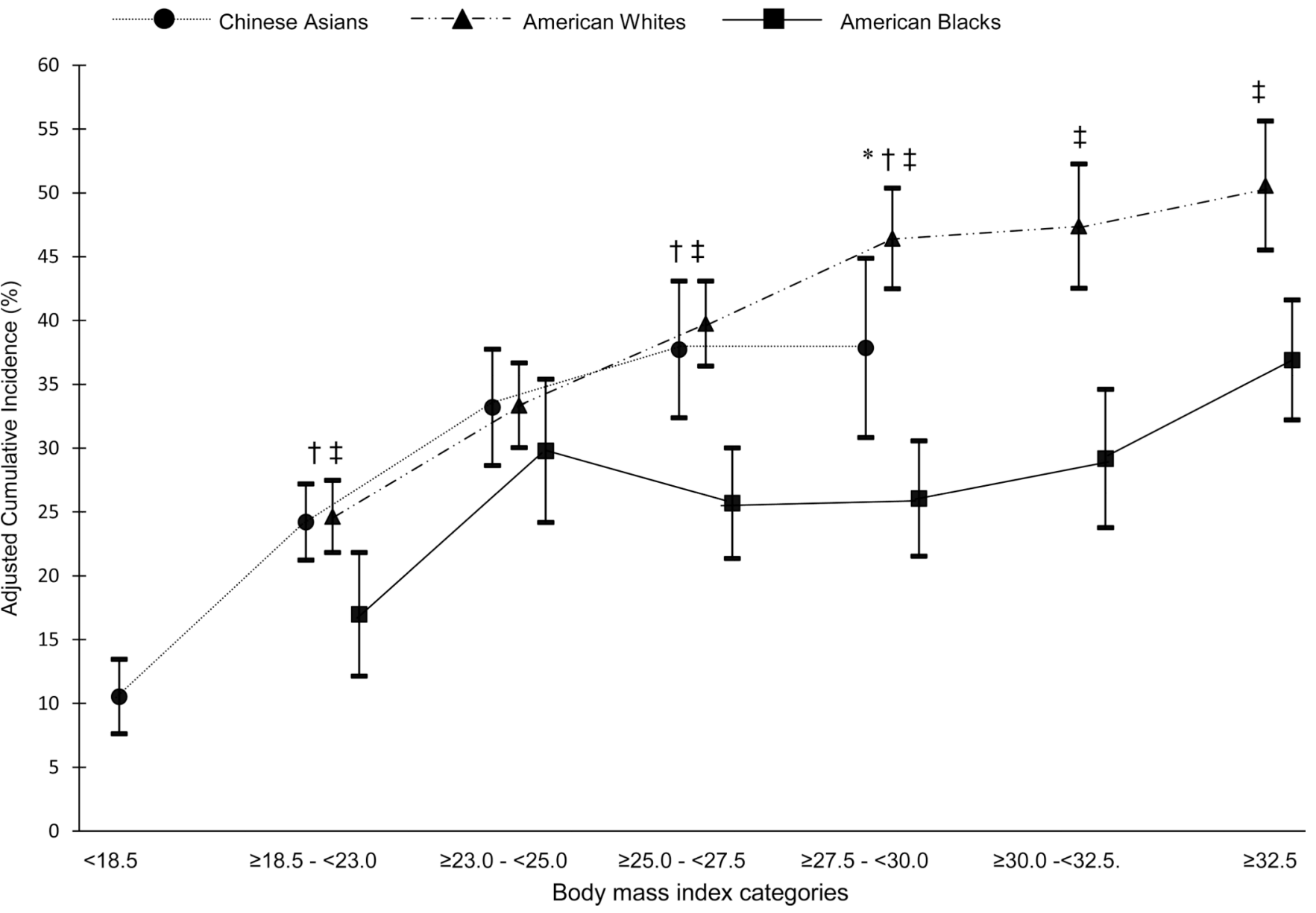

Atherosclerosis. Author manuscript; available in PMC 2012 October 1. 
$1 d$

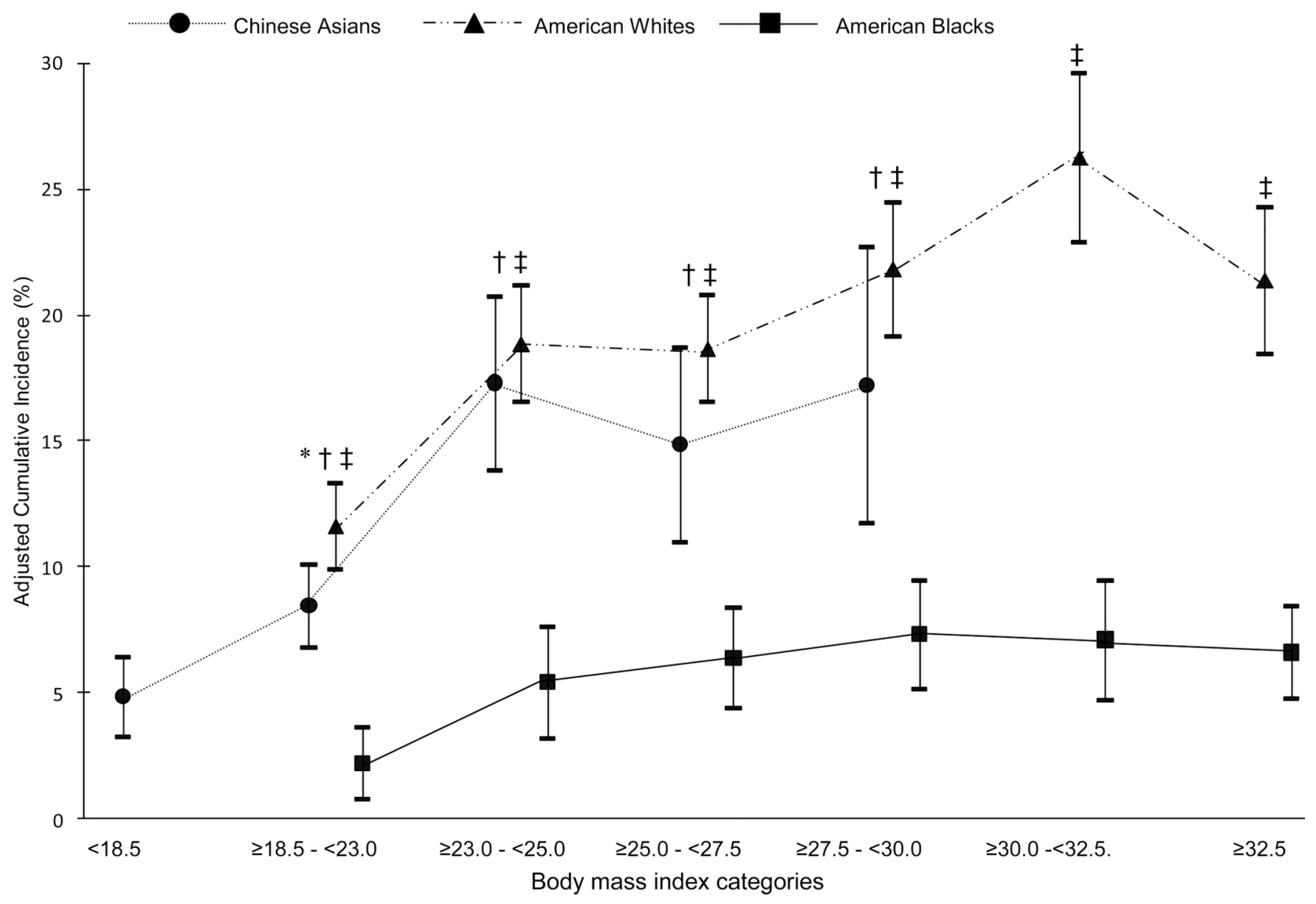

Figure 1.

Adjusted cumulative incidence (and 95\% confidence interval) for (A) high total cholesterol ( $\geq 240 \mathrm{mg} / \mathrm{dL}$ ), (B) high LDL-cholesterol ( $\geq 160 \mathrm{mg} / \mathrm{dL}$ ), (C) low HDL-cholesterol ( $\leq 40 \mathrm{mg} /$ $\mathrm{dL}$ in men and $\leq 50 \mathrm{mg} / \mathrm{dL}$ in women), and (D) high triglycerides ( $\geq 200 \mathrm{mg} / \mathrm{dL}$ ) by BMI categories and ethnicity, the Atherosclerosis Risk in Communities (ARIC) Study, 19871998 and the People's Republic of China (PRC) Study, 1983-1994. Models were adjusted for age, gender, field center, education, smoking status and drinking status. Point estimates for the same BMI categories are slightly shifted in the horizontal plane so that confidence intervals are clearly visible. ${ }^{*}$ Chinese Asians and American Whites are significantly different $(\mathrm{p}<0.05) ; \dagger$ Chinese Asians and American Blacks are significantly different $(\mathrm{p}<0.05) ; \ddagger$ American Whites and American Blacks are significantly different $(\mathrm{p}<0.05)$. 
2a

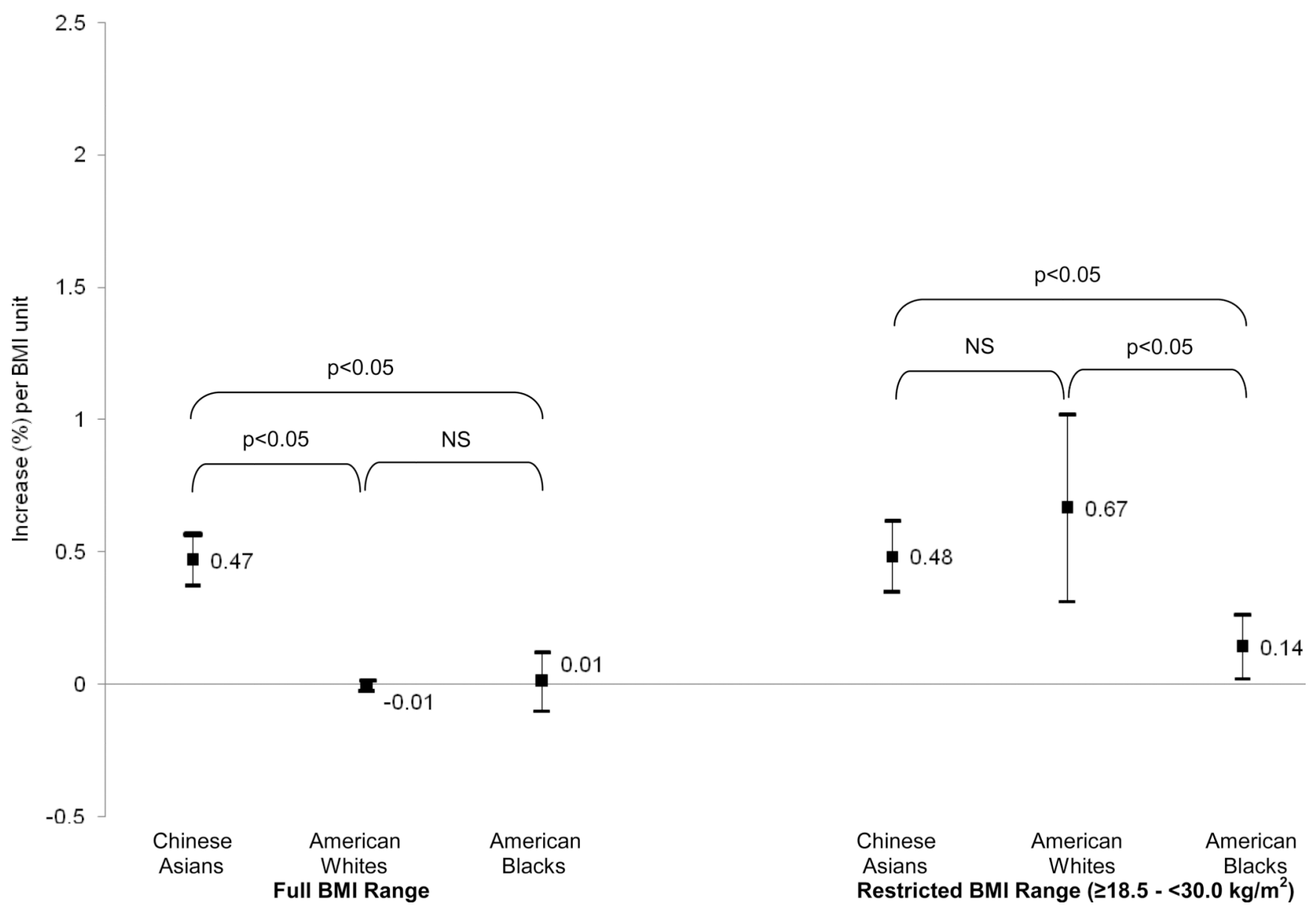




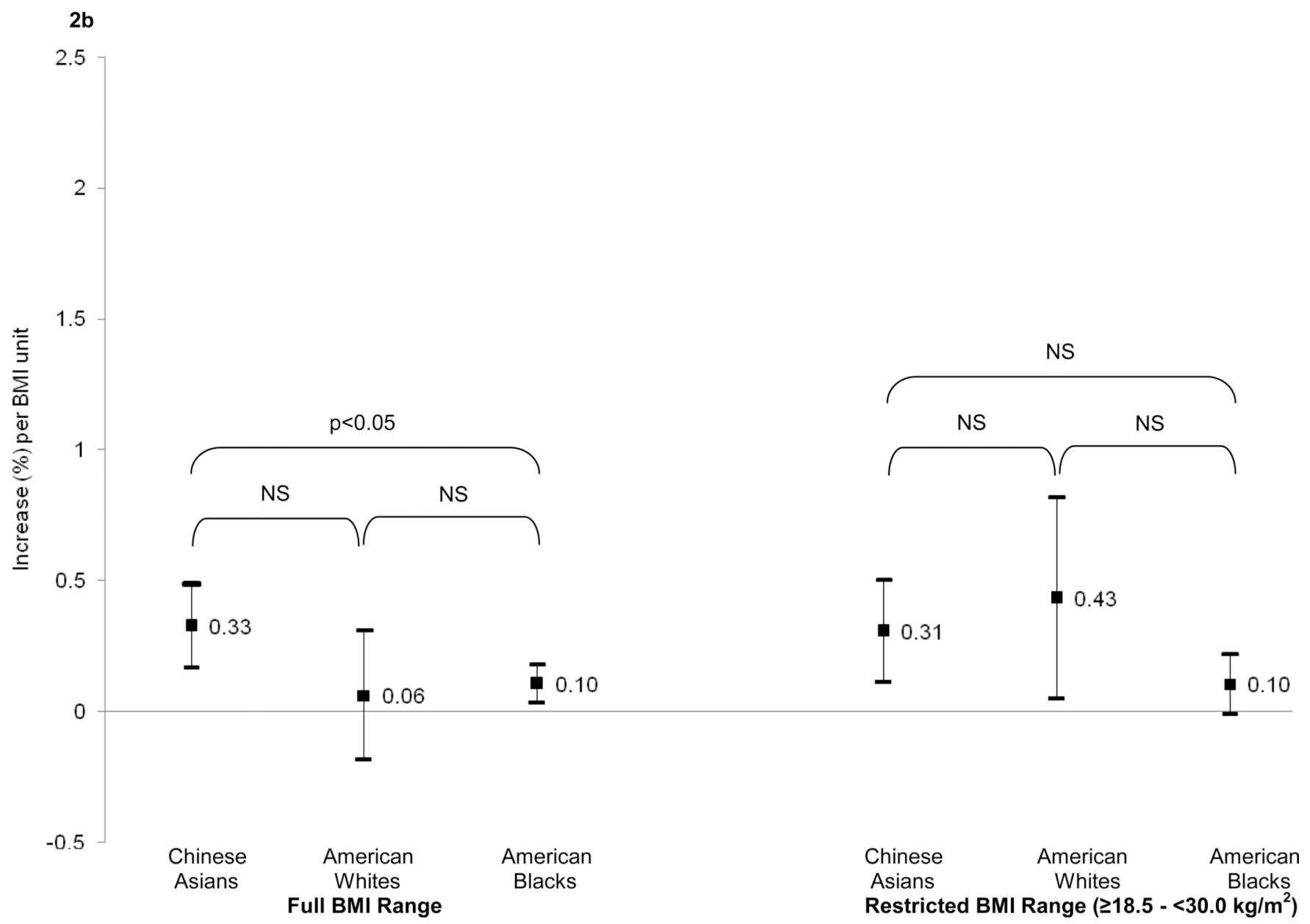


2c

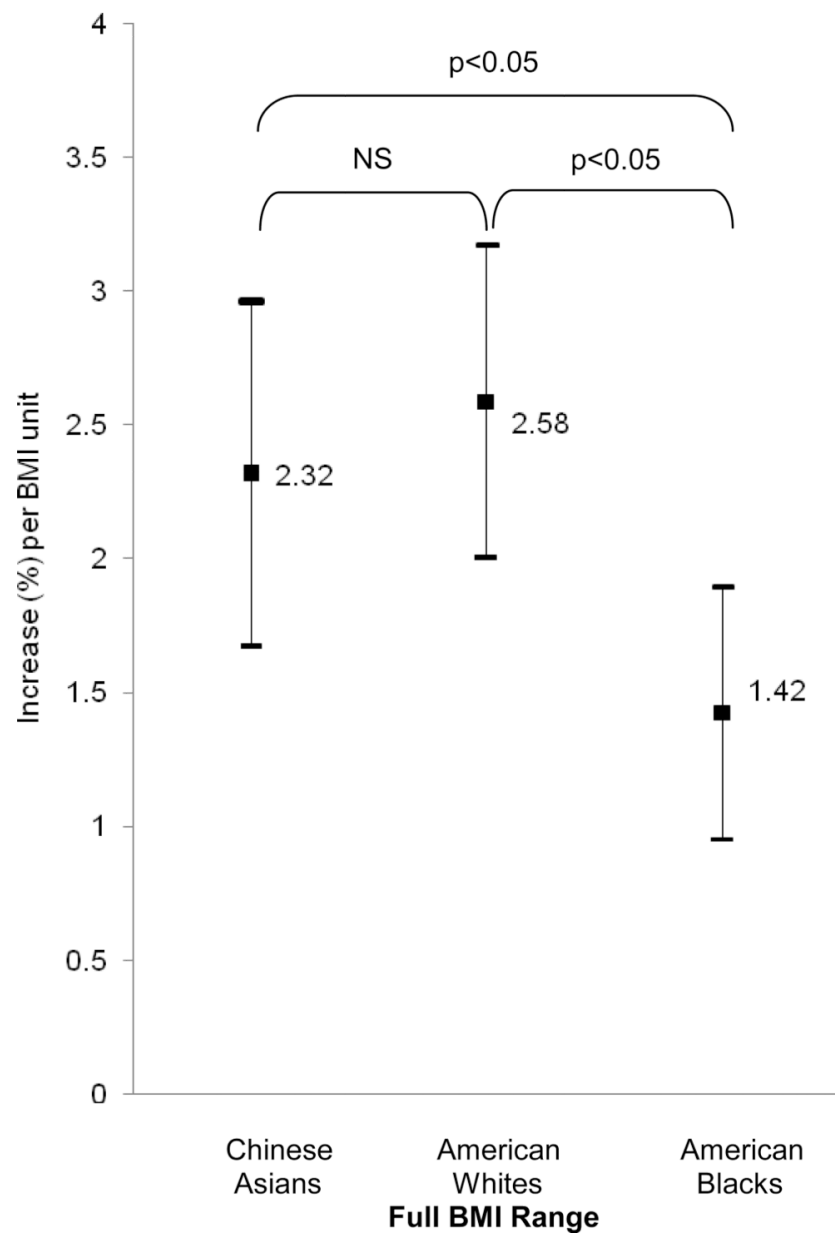

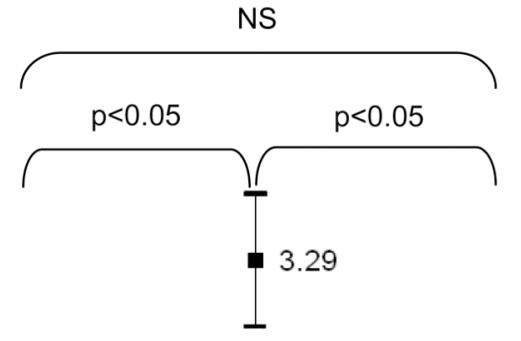
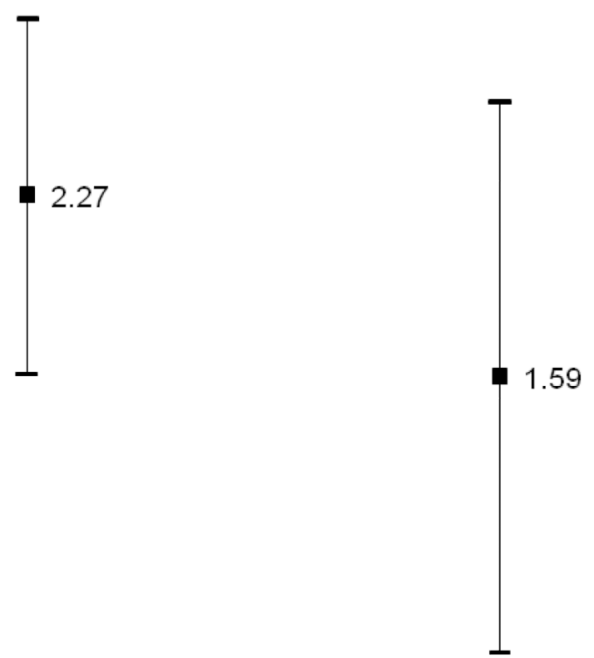

Chinese

Asians

American

Whites

Restricted BMI Range ( $\left.\geq 18.5-<30.0 \mathrm{~kg} / \mathrm{m}^{2}\right)$ 


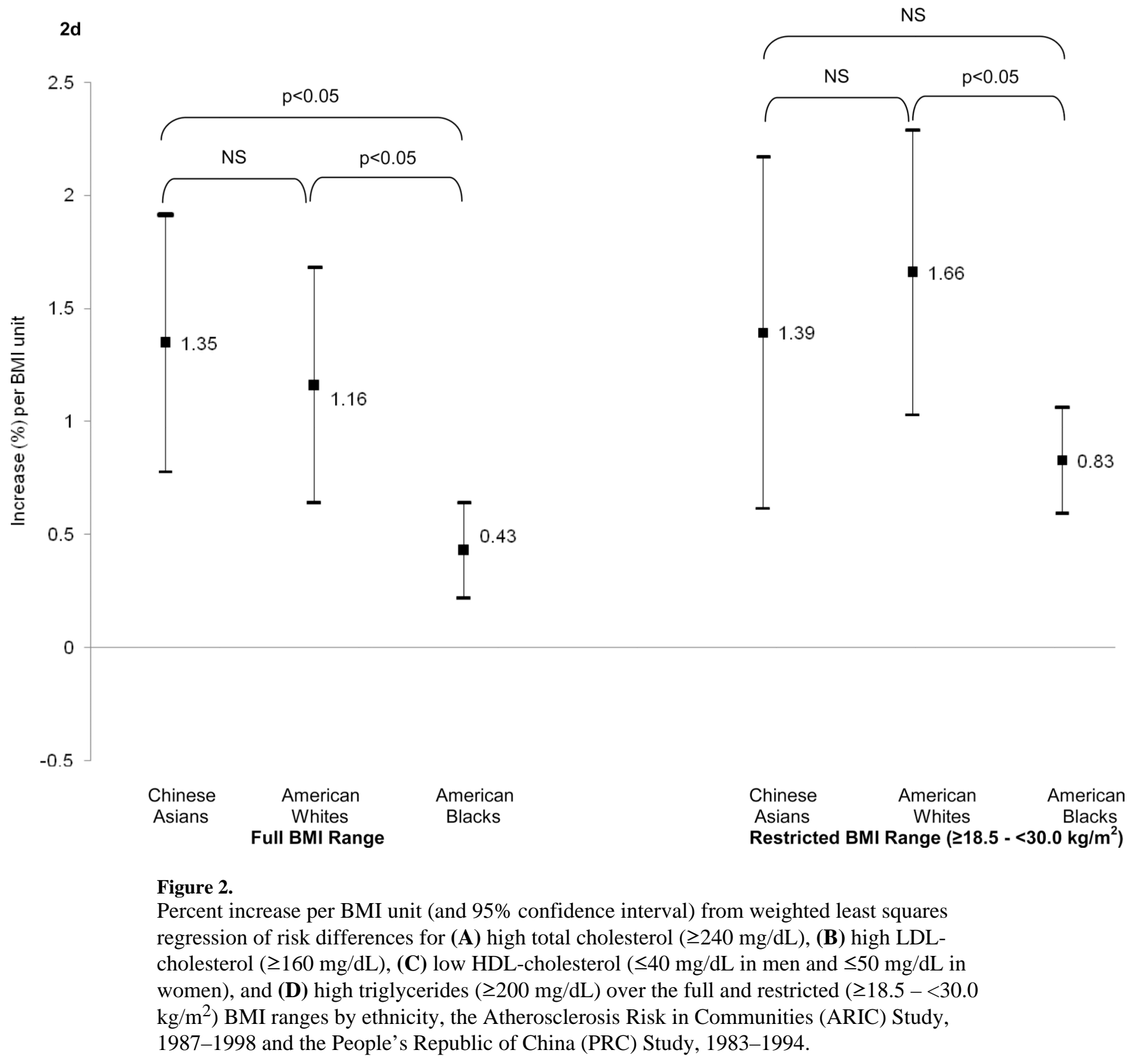

Atherosclerosis. Author manuscript; available in PMC 2012 October 1. 
TABLE 1

Baseline demographic characteristics among Chinese Asians, American Whites and American Blacks, the Atherosclerosis Risk in Communities (ARIC) Study, 1987-1998 and the People's Republic of China (PRC) Study, 1983-1994

\begin{tabular}{|c|c|c|c|}
\hline & $\begin{array}{c}\text { Chinese } \\
\text { Asians } \\
\mathrm{N}=5,303\end{array}$ & $\begin{array}{c}\text { American } \\
\text { Whites } \\
\mathbf{N}=\mathbf{1 0 , 7 5 2}\end{array}$ & $\begin{array}{c}\text { American } \\
\text { Blacks } \\
\mathbf{N}=\mathbf{3 , 4 0 8}\end{array}$ \\
\hline Age (years, mean $\left.\left(\mathrm{SD}^{\dagger}\right)\right)$ & $50.5(3.8)$ & $54.3(5.7)$ & $53.4(5.8)$ \\
\hline Gender ( $\%$ female) & 50.6 & 53.0 & 62.6 \\
\hline Baseline body mass index $\left(\mathrm{kg} / \mathrm{m}^{2}\right.$, mean $\left.(\mathrm{SD})\right)$ & $22.4(3.6)$ & $27.0(4.8)$ & $29.6(6.1)$ \\
\hline \multicolumn{4}{|l|}{ Baseline body mass index $\left(\mathrm{kg} / \mathrm{m}^{2}\right)$ category $(\%)$} \\
\hline$<18.5$ & 13.3 & 0.8 & 0.8 \\
\hline$\geq 18.5-<23.0$ & 46.7 & 19.3 & 9.0 \\
\hline$\geq 23.0-<25.0$ & 17.4 & 17.3 & 11.5 \\
\hline$\geq 25.0-<27.5$ & 13.2 & 23.4 & 19.5 \\
\hline$\geq 27.5-<30.0$ & 6.8 & 16.8 & 18.7 \\
\hline$\geq 30.0-<32.5$ & 2.0 & 10.6 & 14.5 \\
\hline$\geq 32.5$ & 0.6 & 11.9 & 25.8 \\
\hline Cigarette or leaf smoking status (\% current) & 48.1 & 26.3 & 30.0 \\
\hline Alcoholic beverage consumption status ( $\%$ current) & 27.3 & 65.2 & 31.5 \\
\hline Educational Attainment (\% high) & 17.1 & 47.3 & 38.8 \\
\hline \multicolumn{4}{|l|}{ Baseline prevalence $(\%)$} \\
\hline High total cholesterol $^{a}$ & 7.7 & 24.2 & 27.1 \\
\hline High LDL-cholesterol $^{b}$ & 7.3 & 25.4 & 27.8 \\
\hline Low HDL-cholesterol ${ }^{c}$ & 25.3 & 41.5 & 32.9 \\
\hline High triglycerides $d$ & 7.9 & 15.2 & 7.6 \\
\hline \multicolumn{4}{|l|}{${ }^{\dagger} \mathrm{SD}$, standard deviation } \\
\hline \multicolumn{4}{|l|}{$\begin{array}{l}a_{\text {High total cholesterol was defined as } \geq 240 \mathrm{mg} / \mathrm{dL} \text {. }} \\
b \text { High LDL-cholesterol was defined as } \geq 160 \mathrm{mg} / \mathrm{dL} \text {. }\end{array}$} \\
\hline
\end{tabular}




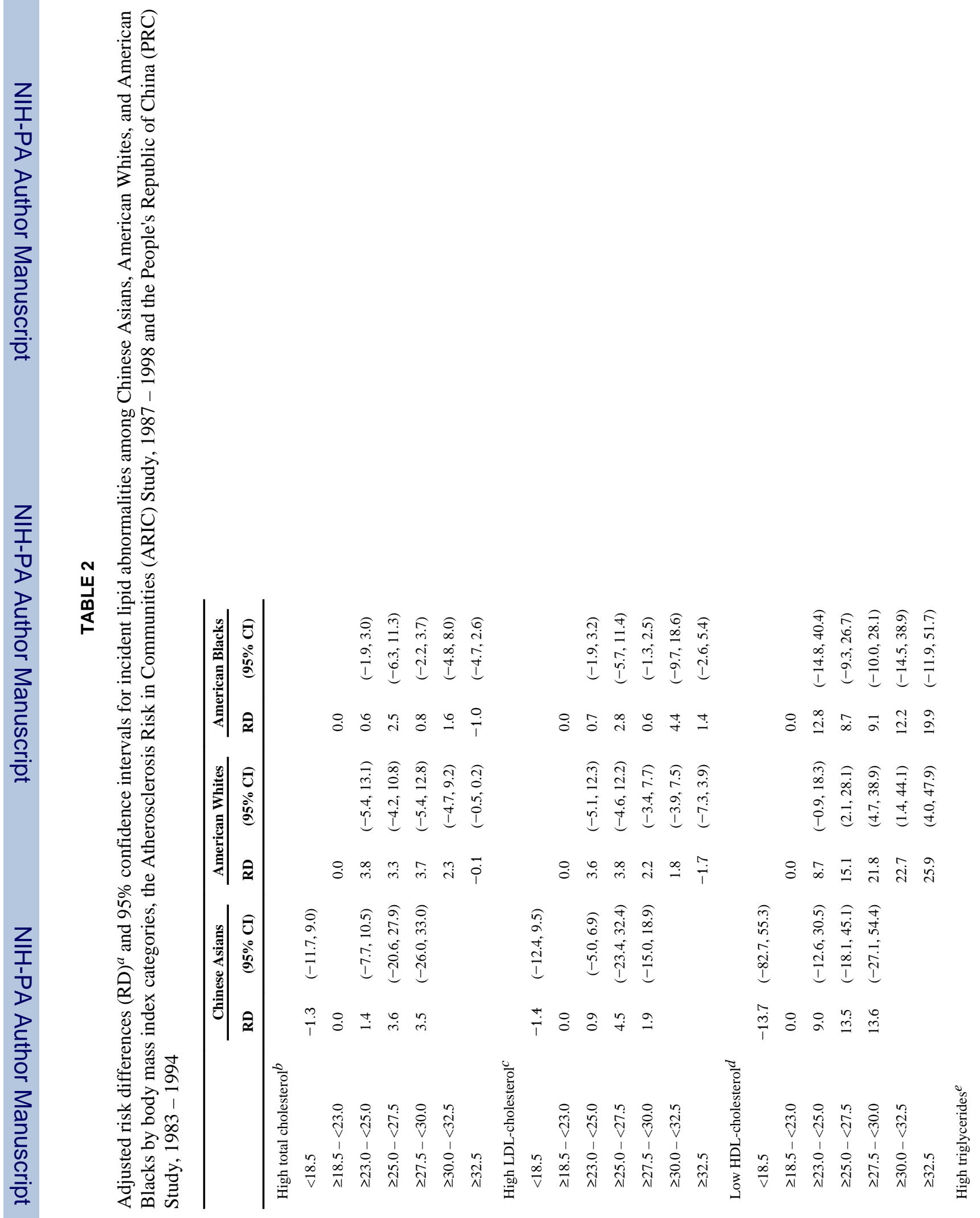


